\title{
Patches Approach to Investigate the Populational Dynamics in Dengue
}

\author{
F.L.P. SANTOS
}

Received on December 10, 2015 / Accepted on February 6, 2017

\begin{abstract}
In areas where resources are located in patches or discrete locations, human dispersal is more conveniently modeled, in which the population is divided into discrete patches. In this work we develop a general discrete model to analyze the spread of Dengue disease. In the process of mathematical modeling we take into account the human populations and the circulation of a single serotype of dengue mosquitoes. The movements of susceptible, infected and recovered humans among all patches are considered. Aquatic phases with different carrying capacities are considered within the patches. Also an arbitrary number of patches can be used to simulate the spread of dengue disease. In this paper we performed numerical experiments to show the applicability of this methodology to investigate the dengue disease problem. The general discrete space model was developed for solving epidemiological problems whereas the humanvector interactions and human mobilities play an important role. Based on our numerical results, we may recommend the general patches model for solving epidemiological problems in Population Dynamics.
\end{abstract}

Keywords: human dispersal, discrete space model, pathway approach, system of ODEs, Euler method, population dynamics.

\section{INTRODUCTION}

Dengue is a febrile infectious disease caused by a virus of the family Flaviridae. It is transmitted to humans by the bite of female mosquitoes, usually of the genus Aedes aegypti. The mosquitoes lives in urban habitats and in man-made containers as a breeding sites in tropical and sub-tropical areas of the world. Due to no improvements in hygiene, sanitation and vector control, containment of dengue disease remains one of the biggest challenges and has been a major public health problem of the modern world, especially in tropical countries such as Brazil, where its incidence has increased in recent years. Brazil reported over 1.5 million cases in 2015, it is approximately three times higher than in 2014, [12]. The infection may develop a lethal complication called Dengue Haemorrhagic Fever, causing serious illnesses and death among children in some Asian and Latin American countries. There are 4 distinct serotypes of the virus that cause dengue (DEN-1, DEN-2, DEN-3 and DEN-4). Dengue vaccine has been licensed for use to people living in endemic areas, but Aedes aegypti also transmits yellow fever, Chikungunya and Zika

Instituto de Biociências de Botucatu, IBB, Departamento de Bioestatística - UNESP, 18618-970 Botucatu, SP, Brasil. E-mail: flpio@ibb.unesp.br 
virus infections. Dengue is a subject of intense research. The simple approach for the analysis of the spread of infectious diseases was initially studied in $[1,2,3]$. In this approach, the population in which a pathogenic agent is active can be divided into three distinct compartments corresponding to different epidemiological status: Susceptible $(S)$, Infected $(I)$ and Recovered $(R)$, i.e., the classical SIR model. Removed individuals can be return to the susceptible compartment and then the SIRS is obtained. Due to the relationship between the movement of populations and the spread of infectious diseases [10], the importance of increased human mobility cannot be underestimated. In [4] the authors claim that human population has a major role in dengue transmission, due to its potential human-aegypti contact. The model involves the dynamic interactions between humans and mosquitoes and takes into account human mobility as an important factor of disease spread. The capacity of human movement across urban areas is the important feature of the urban dengue to propagate infection spatially $[4,5,6]$. Humans are the main amplifying host of the virus. Dengue virus circulating in the blood of viraemic humans is ingested by female mosquitoes during feeding. The virus then infects the mosquito midgut and subsequently spreads systemically over a period of 8-12 days. After this extrinsic incubation period, the virus can be transmitted to other humans during subsequent probing or feeding [12]. In this paper, we describe discrete patches methodology to analize the dynamic of the dengue disease where the movements of human populations has an important role.

\section{MATHEMATICAL EPIDEMIC MODEL}

Due to population dispersal an epidemic model was proposed to describe the dynamics of disease spread among patches in [13]. In [14] the authors considered two-patches where the individuals can move from one patch to another. But the interactions are governed by a classical SIRS model. Extending the classical mathematical epidemic model using pathways, we analyzed how different transmission pathways affect the dynamic of the dengue disease. Due to this, a general methodology is proposed where an arbitrary number of patches with aquatic phase compartments can be considered, as an extension for the classical mathematical model SIR for humans and SI for vector populations and their interactions, $[16,17]$.

Table 1: States variables definition for the model (2.1).

\begin{tabular}{ll}
\hline$A$ & Aquatic phase \\
$S_{h}$ & Susceptible human density \\
$I_{h}$ & Infected human density \\
$R_{h}$ & Recovered human density \\
$S_{v}$ & Susceptible vector density \\
$I_{v}$ & Infected vector density \\
\hline
\end{tabular}

The state variables definition for the proposed mathematical model using patches approach can be found in Table 1. The respective parameters are shown in Table 2. Figure 1 show the diagram of the dynamic population of the general model considering an arbitrary number $k$ of patches. 
Table 2: Biological parameters for the model (2.1).

\begin{tabular}{ll}
\hline$\mu_{A}$ & per capita aquatic mortality rate \\
$\mu_{h}$ & per capita human natality and mortality rates \\
$\mu_{v}$ & per capita mosquito mortality rate \\
$C$ & carrying capacity related to the amount of available nutrients and space \\
$\phi$ & is the intrinsic oviposition rate \\
$\alpha$ & proportion of mosquito becoming susceptible \\
$b$ & proportion of the effective bite that transmit infection \\
$\gamma$ & recovery rate of humans \\
$\beta_{h}$ & vector-human probability of transmission \\
$\beta_{v}$ & human-vector probability of transmission \\
$N_{h}$ & Total number of humans \\
$K_{i j}$ & humans movement rate between compartment $i$ to $j$ \\
\hline
\end{tabular}

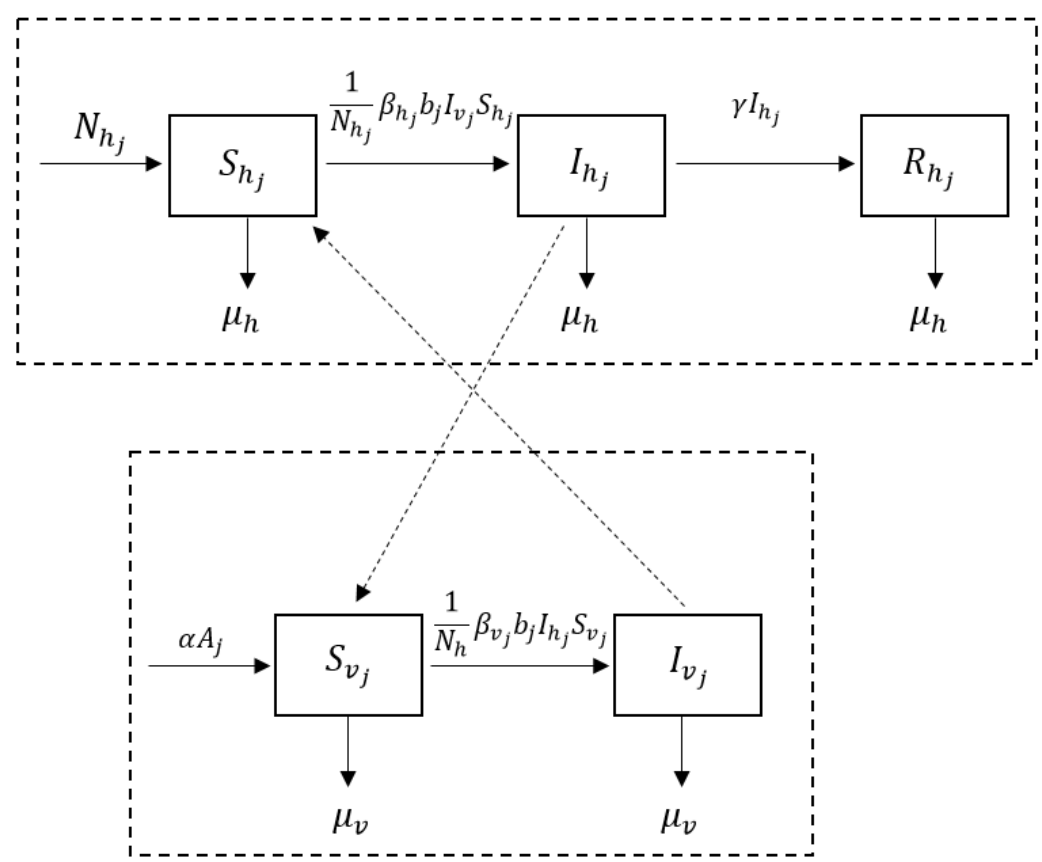

Figure 1: Compartmental diagram of dynamic interactions of general epidemiological model.

Considering all assumptions, the corresponding model is given by the system (2.1) below, together with $N_{h j}=S_{h j}+I_{h j}+R_{h j}$, for $j=0, \ldots, k$, where $k$ is the number of patches. 


$$
\left\{\begin{array}{l}
\frac{d A_{j}}{d t}=\phi\left(1-\frac{A_{j}}{C_{j}}\right)\left(S_{v j}+I_{v j}\right)-\left(\alpha+\mu_{A}\right) A_{j} \\
\frac{d S_{h j}}{d t}=\mu_{h}\left(N_{h j}-S_{h j}\right)-\frac{1}{N_{h}} \beta_{h j} b_{j} I_{v j} S_{h j}+\sum_{j=0}^{k} K_{j l} S_{h l}-\sum_{j=0}^{k} K_{l j} S_{h j}, \\
\frac{d I_{h j}}{d t}=\frac{1}{N_{h}} \beta_{h j} b_{j} I_{v j} S_{h j}-\left(\mu_{h}+\gamma\right) I_{h j}+\sum_{j=0}^{k} K_{j l} I_{h l}-\sum_{j=0}^{k} K_{l j} I_{h j}, \\
\frac{d R_{h j}}{d t}=\gamma I_{h j}-\mu_{h} R_{h j}+\sum_{j=0}^{k} K_{j l} R_{h l}-\sum_{j=0}^{k} K_{l j} R_{h j} \\
\frac{d S_{v j}}{d t}=\alpha A_{j}-\frac{1}{N_{h}} \beta_{v j} b_{j} I_{h j} S_{v j}-\mu_{v} S_{v j} \\
\frac{d I_{v j}}{d t}=\frac{1}{N_{h}} \beta_{v j} b_{j} I_{h j} S_{v j}-\mu_{v} I_{v j}
\end{array}\right.
$$

for all $l=0, \ldots, k(l \neq j)$. To obtained the numerical solutions, the system (2.1) was discretized by the following scheme, as described in next section.

\section{DISCRETIZATION}

To obtain the numerical results, the system (2.1) and in human mobility terms were discretized by the following scheme in relation to the derivatives at the time $t$.

\subsection{Time-discretization}

In relation to time discretization of the derivative terms, the scheme is constructed by the procedure as described in the following two main steps:

1. the classical forward Euler integration method is employed for the time discretization of the derivative at the left-hand side of the system (2.1); For example, in a general function $f$, the following fully discrete method for $t>0$ is given by:

$$
\frac{d}{d t} f=\frac{f(t+d t)-f(t)}{d t}+O(d t)
$$

where $d t=T / N$ is the time-step, $T$ is total time of simulation, $N$ is the number of points of the discretization and $O(d t)$ is the error order of the scheme.

2. all linear terms at the right-hand side of the system (2.1) are replaced by discrete representation at the forward time $t+d t$.

Following steps 1 and 2, for example in the continuous equation of aquatic phase $A$, we obtain the corresponding discretized form:

$$
\frac{A_{i+1, j}-A_{i, j}}{d t}=\phi\left(1-\frac{A_{i, j}}{C_{j}}\right)\left(S_{v i, j}+I_{v i, j}\right)-\left(\alpha+\mu_{A}\right) A_{i+1, j}
$$


The main reason for replacing only the right-hand side of Eq. (2.1) is the possibility to obtain the implicit numerical method with a very simple strategy, i.e, when we adopt this procedure, only the trivial algebra is required to isolate all state variables at time $i+1$, as described in system (3.3). In addition, this numerical method is very easy to implement computationally as it is accurate in terms of obtaining numerical results in the performed tests, involving a patch environment. Higher-order discretization schemes can be considered, but they do not show any improvements in the results. Finally, this method can be an alternative approach to the classical ones, having the potential to be used in Computational Population Dynamics problems.

Thus, following the two steps as described above in each equations of the system (2.1) and after a simple algebra, we can finally obtain the corresponding discretized system as below:

$$
\left\{\begin{array}{l}
A_{i+1, j}=\frac{1}{1+d t\left(\alpha+\mu_{A}\right)}\left(A_{i, j}+d t \phi\left(1-\frac{A_{i, j}}{C_{j}}\right)\left(S_{v i, j}+I_{v i, j}\right)\right) \\
S_{h i+1, j}=\frac{1}{1+d t \mu_{h}}\left(S_{h i, j}+d t\left(\mu_{h} N_{h j}-\frac{1}{N_{h j}} b_{j} \beta_{h j} I_{v i, j} S_{h i, j}+\sum_{i n} S_{j}-\sum_{\text {out }} S_{j}\right)\right) \\
I_{h i+1, j}=\frac{1}{1+d t\left(\mu_{h}+\gamma_{j}\right)}\left(I_{h i, j}+d t\left(\frac{1}{N_{h j}} b_{j} \beta_{h j} I_{v i, j} S_{h i, j}+\sum_{i n} I_{j}-\sum_{\text {out }} I_{j}\right)\right) \\
R_{h i+1, j}=\frac{1}{1+d t \mu_{h}}\left(R_{h i, j}+d t\left(\gamma_{j} I_{h i, j}+\sum_{i n} R_{j}-\sum_{\text {out }} R_{j}\right)\right) \\
S_{v i+1, j}=\frac{1}{1+d t \mu_{v}}\left(S_{v i, j}+d t\left(\alpha A_{i, j}-\frac{1}{N_{h j}} b_{j} \beta_{v j} I_{h i, j} S_{v i, j}\right)\right) \\
I_{v i+1, j}=\frac{1}{1+d t \mu_{v}}\left(I_{v i, j}+d t\left(\frac{1}{N_{h j}} b_{j} \beta_{v j} I_{h i, j} S_{v i, j}\right)\right) \\
N_{h j}=S_{h i+1, j}+I_{h i+1, j}+R_{h i+1, j},
\end{array}\right.
$$

for $i=1, \ldots, N-1$ and $j=1, \ldots, k$. The discretization of human mobility terms is described as follows.

\subsection{Human mobility discretization}

For all $j=1, \ldots, k$ with $l=1, \ldots, k$ and if $j \neq l$, the human mobility effects are incorporated to each human compartment $S_{h}, I_{h}$ and $R_{h}$, by computing:

$$
\left\{\begin{array}{l}
\sum_{\text {in }} S_{j}=\sum_{\text {in }} S_{j}+K_{j, l} S_{h i, l} \\
\sum_{\text {out }} S_{j}=\sum_{\text {out }} S_{j}-K_{l, j} S_{h i, j} \\
\sum_{\text {in }} I_{j}=\sum_{\text {in }} I_{j}+K_{j, l} I_{h i, l} \\
\sum_{\text {out }} I_{j}=\sum_{\text {out }} I_{j}+K_{l, j} I_{h i, j} \\
\sum_{\text {in }} R_{j}=\sum_{\text {in }} R_{j}+K_{j, l} R_{h i, l} \\
\sum_{\text {out }} R_{j}=\sum_{\text {out }} R_{j}+K_{l, j} R_{h i, j},
\end{array}\right.
$$


where $K$ is the random matrix of human movement ratios among all compartments. Figure 2 show the dynamic of $K_{i j}$ between compartments $i$ to $j$.

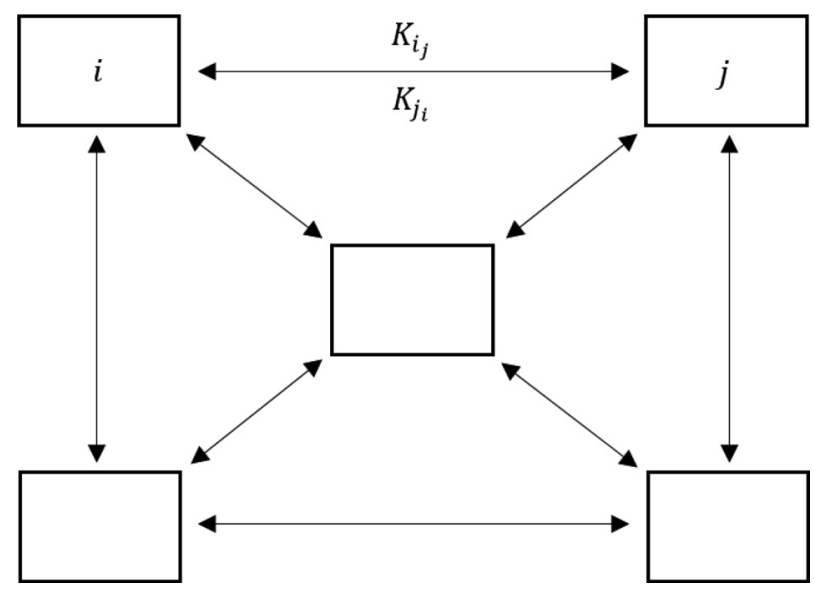

Figure 2: Patches approach and human movement rates $K_{i j}$ between compartments $i$ to $j$.

Next section presents the simulation performed using the general model proposed, where the focus is show the effects of human mobility in the spread of the dengue disease in patch environment.

\section{NUMERICAL SIMULATION}

To show the ability of the model in the dynamic simulation of dengue disease involving $k$ discrete patches, we performed test simulations for $k=1, k=2$ and $k=5$. The respective matrix human movement rate, $K$, are given by a random process. In a particular case test $1, K=0$. For all tests we set $C_{j}=C=$ constant . The parameters used for all simulations are shown in Table 3 .

Table 3: Parameters of simulation $\left(\right.$ days $\left.^{-1}\right)$.

\begin{tabular}{ccccc}
\hline$C$ & $\mu_{A}$ & $\mu_{h}$ & $\mu_{v}$ & $\phi$ \\
\hline $13[15]$ & $0.0583[18]$ & $0.457 \times 10^{-4}[16]$ & $0.25[16]$ & $6.353[18]$ \\
\hline \hline$\alpha$ & $b$ & $\gamma$ & $\beta_{h}$ & $\beta_{v}$ \\
\hline $0.121[18]$ & $1.0[16]$ & $0.167[16]$ & $0.4[16]$ & $0.4[16]$ \\
\hline
\end{tabular}

\subsection{Test 1: $k=1$}

The dynamic of model show in this test that in the presence of the human infected in the patch, the mosquito susceptible population becomes infected, as shown in Figure 3. It also shows the low initial density of aquatic phase increasing very fast, as in Figure 4. 


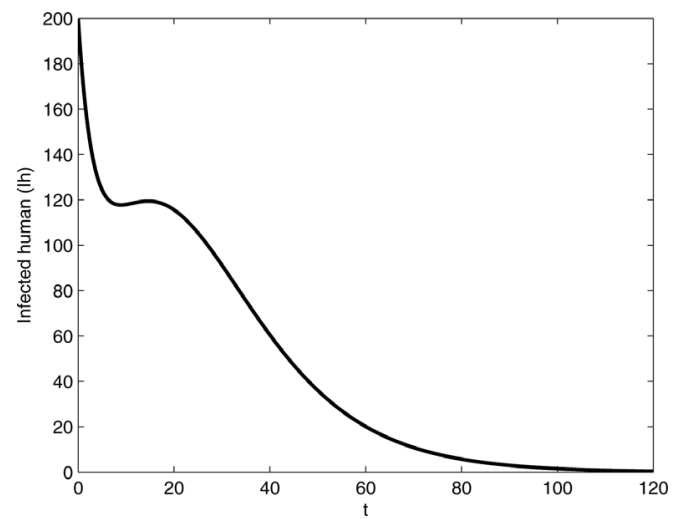

(a) Dynamic of the Infected Human

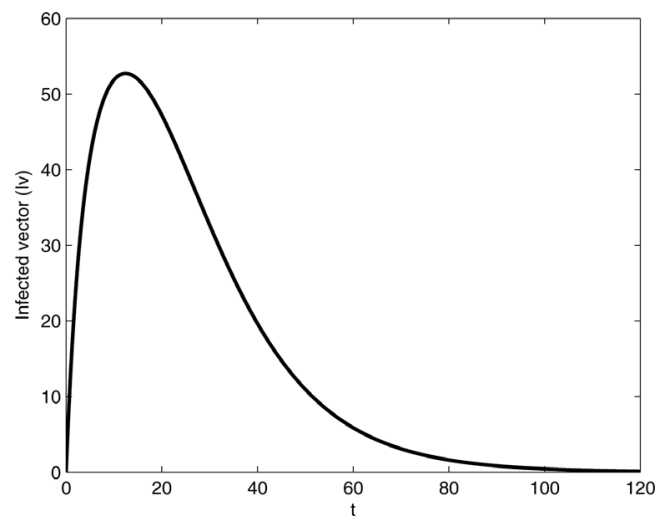

(b) Dynamic of the Infected Mosquitoes

Figure 3: Dynamic of the infected population within of one patch, $k=1$. Initial conditions: (a) $I_{h 1}(0)=200$; (b) $I_{v 1}(0)=0 . T=120$ days.

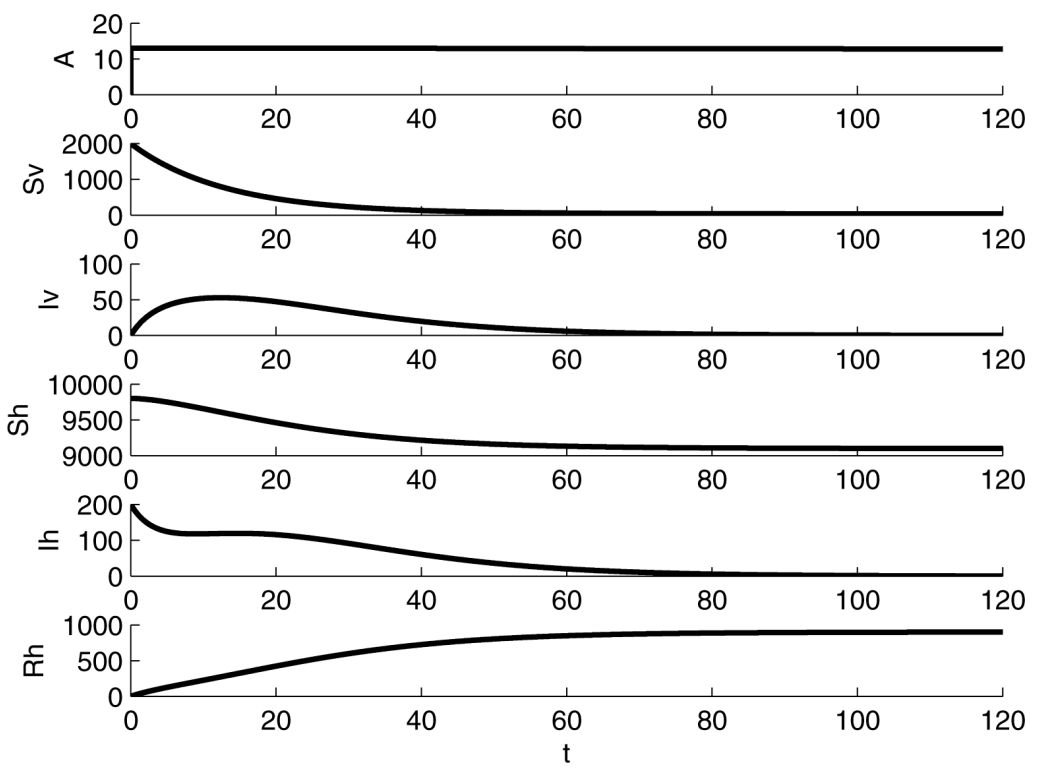

Figure 4: Dynamic population of model using $k=1$. Parameters: $A=0.0001 ; N h=10000$, $I_{h}=200, S_{v}=2000 ; \mathrm{T}=120$ days $; t=0.01 ; K=0$.

\subsection{Test 2: $k=2$}

Figures 5 and 6 show numerical results of the dynamic of all populations considering two patches, $k=2$. In this dynamic, due to the presence of infected mosquitoes in the patch 1 , the human susceptible population became infected in this patch 1 . Because of the human mobility considered 
between the patches, the human infected population can move from 1 to 2 and the susceptible mosquitoes in patch 1 become infected. Finally, the human population infected increased in the patch 2. In order to show the ability of the general epidemiological model in the simulation for an arbitrary $k$ number of discrete patches, we performed the simulation considering $k=5$ patches, as following.

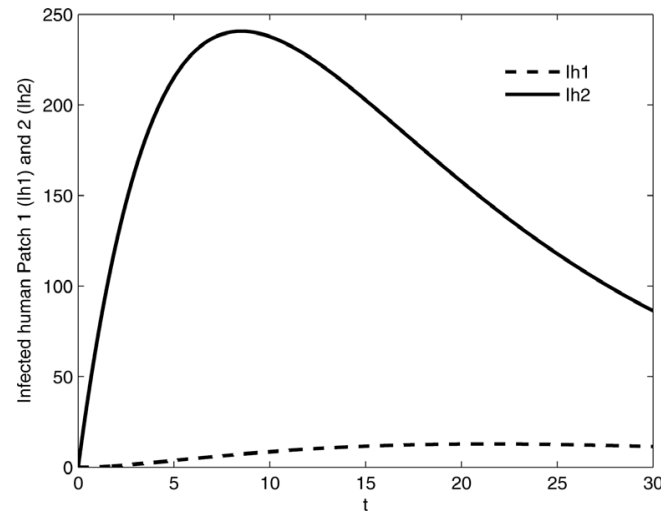

(a) Dynamic of the Infected Humans

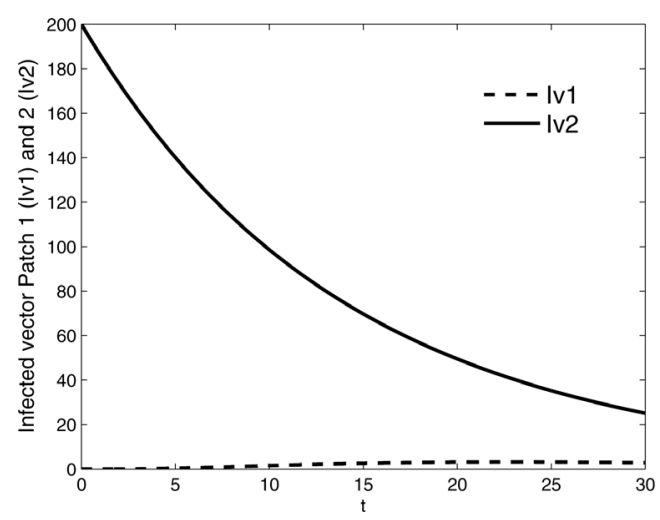

(b) Dynamic of the Infected Mosquitoes

Figure 5: Dynamic of the infected population within the patches 1 and 2. $T=30$ days. Initial conditions: (a) $I_{h 1}(0)=I_{h_{2}}(0)=0$. (b) $I_{v 1}(0)=200$ and $I_{v 2}(0)=0$.
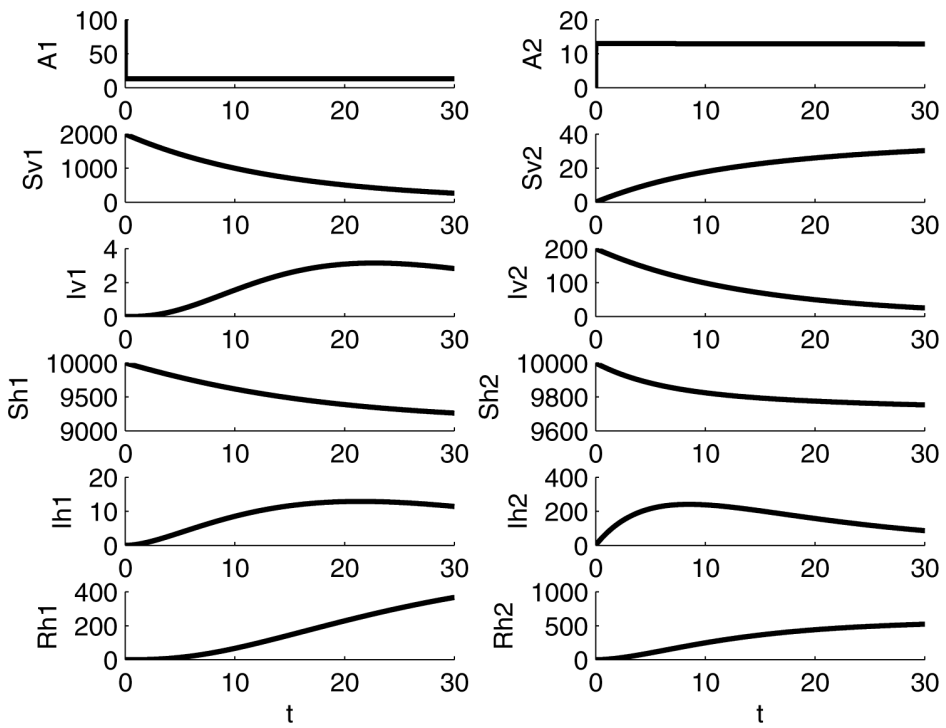

Figure 6: Dengue disease spread considering two patches, $k=2$. Parameters: $A_{1}(0)=100$, $A_{2}(0)=0.0001, S_{v 1}(0)=2000 ; N_{h 1}=N_{h 2}=10000 ;$ Total time T= 30 days; Time-step $d t=0.01$. 


\subsection{Test 3: $k=5$}

The initial conditions for this test can be see in the following Table 4. Figures 7(a) and (b) show the dynamics of human and mosquito infected populations, respectively. Figure 8 shows the dynamic behavior of all populations within all patches. It is important to note the effects of human mobility among all five patches and their impact on the infection in the susceptible populations.

Table 4: Initial conditions for the model for all patches $j=1, \ldots, k=5$.

\begin{tabular}{llllll}
\hline & 1 & 2 & 3 & 4 & 5 \\
\hline$A$ & 100 & 0 & 0 & 100 & 0.0001 \\
$S_{v}$ & 2000 & 0 & 2000 & 2000 & 0 \\
$I_{v}$ & 0 & 0 & 10 & 0 & 0 \\
$S_{h}$ & 90000 & 100000 & 100000 & 100000 & 100000 \\
$I_{h}$ & 2000 & 0 & 0 & 0 & 0 \\
$R_{h}$ & 0 & 0 & 0 & 0 & 0 \\
\hline
\end{tabular}

In the next section, the main conclusions of this work are drawn, where a general epidemiological model using patch approaches was developed to explain how the human mobility can affect the spread of dengue disease.

\section{CONCLUSIONS}

The main conclusions that can be drawn are:

1. the methodology proposed here allowed an analysis of the epidemic disease spread using an arbitrary number of patches particularly in dengue;

2. the effects of human mobilities among all patches was incorporated in this general epidemiological model;

3. aquatic phase compartment with different carrying capacities can also be considered in these patch approaches;

4. due to the infected human populations mobility the susceptible mosquito population became infected in any patches considered;

5. the numerical method used to solve the model in patches environment showed to be an alternative approach in Computational Population Dynamics problems.

Based on our results, we may recommend the general patches model for solving epidemiological problems where the vector-human interactions and human mobilities have important roles. 


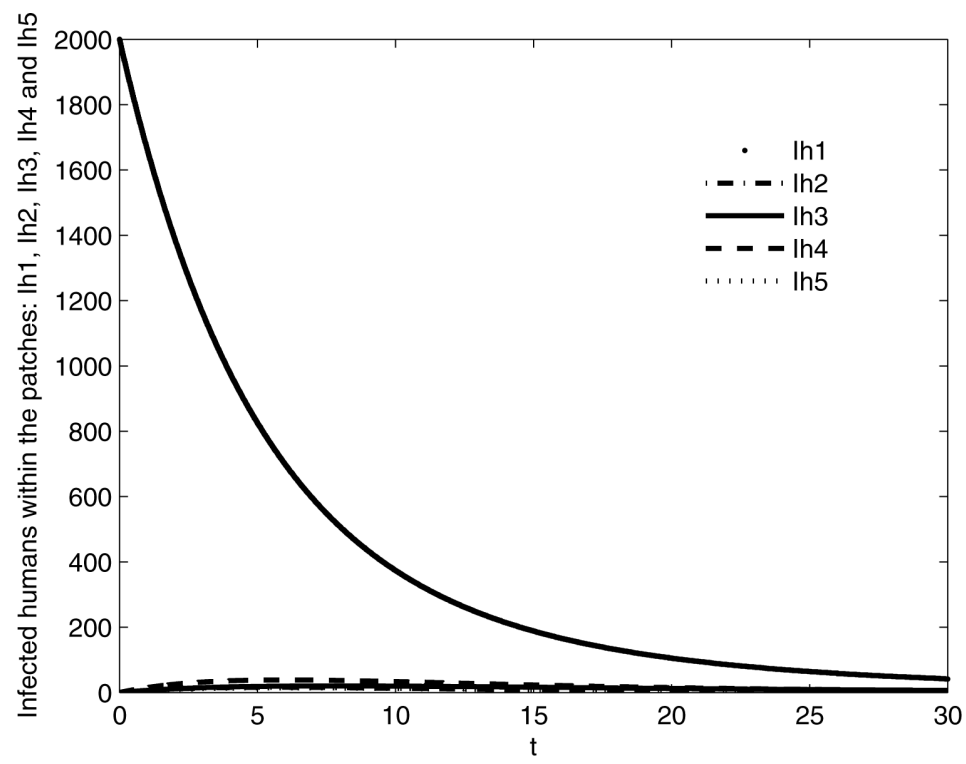

(a) Dynamic of the Infected Humans

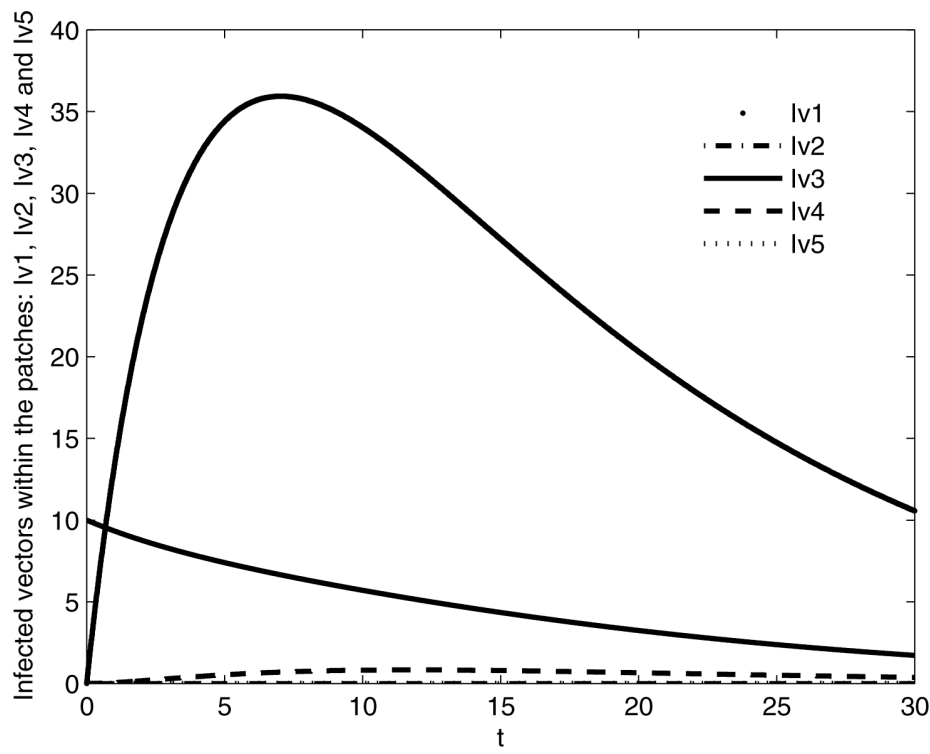

(b) Dynamic of the Infected Mosquitoes

Figure 7: Dynamic of the infected populations within all the patches. $k=5$.

\section{ACKNOWLEDGMENTS}

The author would like to thank the Professors Claudia Pio Ferreira (Institute of Biosciences of Botucatu, IBB/Unesp) and Bedr'Eddine Ainseba (Institute of Mathematical of Bordeaux, IMB) for insightful discussions and suggestions. 

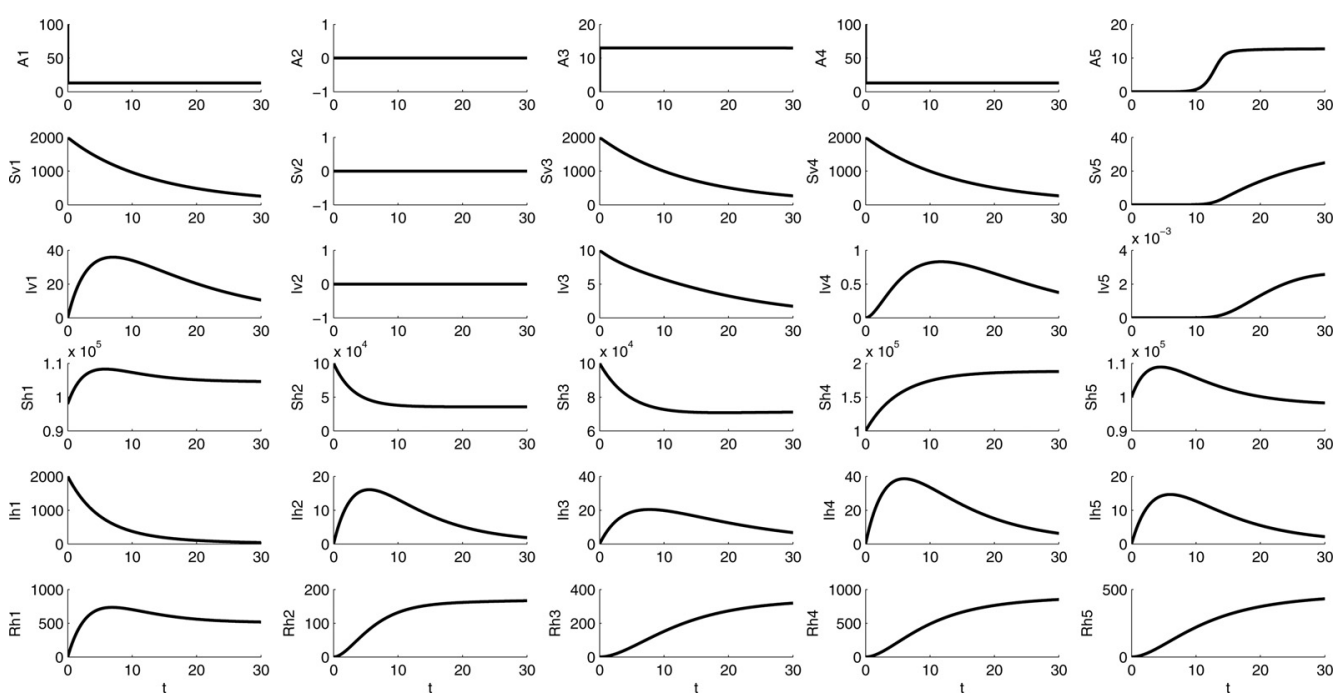

Figure 8: Dengue disease spread considering $k=5$ patches. Parameters: Total time: $\mathrm{T}=30$ days; Time-step: $d t=0.01$.

RESUMO. Em áreas onde os recursos estão localizados em locais discretos, a dispersão humana é mais convenientemente modelada considerando a população dividida em regiões discretas (patches). Neste trabalho, desenvolvemos um modelo espaço discreto geral para analisar a propagação da doença da dengue. No processo de modelagem matemática, levamos em consideração as populações humanas e a circulação de um único sorotipo de mosquitos da dengue. A mobilidade dos seres humanos suscetíveis, infectados e recuperados entre todos os patches é considerada. As fases aquáticas com distintas capacidades de suporte são consideradas dentro de cada patch. Além disso, um número arbitrário de patches podem ser usados para simular a propagação da doença da dengue. Este modelo espacialmente discreto foi desenvolvido para resolver problemas epidemiológicos, em que interações homemvetor e a mobilidade humana desempenham um papel importante. Com base nos resultados numéricos obtidos, podemos recomendar o modelo em patches para a resolução de problemas epidemiológicos em Dinâmica Populacional.

Palavras-chave: Dispersão humana, modelo espaço discreto, abordagem pathway, sistema de EDO’s, método de Euler, dinâmica populacional.

\section{REFERENCES}

[1] W.H. Hamer. Epidemic disease in England. Lancet, 1 (1906), 733-739.

[2] R. Ross. The prevention of malaria, 2nd edn. John Murray, London, (1911). 
[3] W.O. Kermack \& A.G. McKendrick. Contributions to the mathematical theory of epidemics, Part I. Proc. R. Soc. Lond. A, 115 (1927), 700-721.

[4] H. Padmanabha, D. Durham, F. Correa, M. Diuk-Wasser \& A. Galvani. The Interactive Roles of Aedes aegypti Super-Production and Human Density in Dengue Transmission. PLOS Neglected Tropical Diseases, 6(8) (2012).

[5] L.C. de Castro Medeiros, C.A. Castilho, C. Braga, W.V. de Souza \& L. Regis et al. Modeling the dynamic transmission of dengue fever: investigating disease persistence. PLoS Negl. Trop. Dis., 5(1) (2011), e942.

[6] N.A. Honorio, R.M. Nogueira, C.T. Codeco, M.S. Carvalho \& O.G. Cruz et al. Spatial evaluation and modeling of Dengue seroprevalence and vector density in Rio de Janeiro, Brazil. PLoS Negl. Trop. Dis., 3 (2009), e545.

[7] B. Adams \& D.D. Kapan. Man Bites Mosquito: Understanding the Contribution of Human Movement to Vector-Borne Disease Dynamics, 4(8) (2009), e6763.

[8] Yu Jin \& W. Wang. The effect of population dispersal on the spread of a disease. J. Math. Anal. Appl., 308 (2005), 343-364.

[9] V. Belik, T. Geisel \& D. Brockmann. Natural Human Mobility Patterns and Spatial Spread of Infectious Diseases. Physical Review X, 1 (2011), 011001.

[10] D. Chen, B. Moulin \& J. Wu. Analyzing and Modeling Spatial and Temporal Dynamics of Infectious Diseases. (2014) John Wiley \& Sons, Inc.

[11] J. Arino \& K. Khan. Using mathematical modelling to integrate disease surveillance and global air transportation data. (2013) John Wiley \& Sons, Inc.

[12] World Health Organization. Dengue: Guidelines for Diagnosis, Treatment, prevention and control, 2nd edn. Geneva: WHO, (2009).

[13] W. Wang \& X.Q. Zhao. An epidemic model in a patch environment. Mathematical Biosciences, 190 (2004), 97-112.

[14] E. Kouokam, P. Auger, H. Hbid \& M. Tchuente. Effect of the Number of Patches in a Multi-patch SIRS Model with Fast Migration on the Basic Reproduction Rate. Acta Biotheor., 56 (2008), 75-86.

[15] H.O. Florentino, B.F. Bannawart, D.R. Cantane \& F.L.P. Santos. Multiobjective genetic algorithm applied to dengue control. Mathematical Biosciences, 258 (2014), 77-84.

[16] H. Nishiura. Mathematical and Statistical Analyses of the Spread of Dengue. Dengue Bulletin, 30 (2006).

[17] M. Andraud, H. Niel, C. Marais \& P. Beutels. Dynamic Epidemiological Models for Dengue Transmission: A Systematic Review of Structural Approaches. PLoS ONE 7, 11 (2012).

[18] R.C.A. Thomé, H.M. Yang \& L. Esteva. Optimal control of Aedes aegypti mosquitoes by the sterile insect technique and insecticide. Mathematical Biosciences, Elsevier, 223 (2010), 12-23. 